\title{
Modeling the formation of layered, amphiphilic gels
}

Santidan Biswas ${ }^{\mathrm{a}}$, Awaneesh Singh ${ }^{\mathrm{a}}$, Antoine Beziau ${ }^{\mathrm{b}}$, Tomasz Kowalewski ${ }^{\mathrm{b}, *}$, Krzysztof

$$
\text { Matyjaszewski }^{\mathrm{b}, * *}, \text { Anna C. Balazs }{ }^{\mathrm{a}, * * *}
$$

Keywords: Two-layer stackable gels; Dissipative particle dynamics; Atom transfer radical polymerization; Free radical polymerization;

\begin{abstract}
The efficient formulation of layered gels that encompass both hydrophilic and hydrophobic domains could lead to novel materials with a range of vital functionalities. To facilitate the fabrication of these materials, we perform dissipative particle dynamics (DPD) simulations to model the formation of two-layered stackable gels where the gels are incompatible and their respective solvents are immiscible. The bottom layer of the gel is created first and then a solution of new initiators, monomers and cross-linkers is introduced on top of this first layer. These components then undergo living copolymerization to form the second gel layer. We investigate all possible combinations using free radical polymerization (FRP) and controlled/living atom transfer radical polymerization (ATRP) to form the two-
\end{abstract}


layered material. When the lower gel is formed via ATRP, the system forms connected, stacked gel layers. If, however, the lower gel is formed via FRP, the gels do not form interconnected layers. The presence of active radical chain ends in the lower gel grown by ATRP enables the formation of covalent bonds with the upper gel layer. On the other hand, the lack of active radicals in the lower FRP gel leads to a sharp interfacial region, with negligible inter-gel bonds connecting the two layers. Our simulations provide insight into the nanoscale nature of the interface between the gel layers and yield guidelines for forming mechanically robust, "stackable" amphiphilic gels.

\section{Introduction}

The ability to create robust, multi-layered, "stackable" gels, where one layer is effectively "stacked" on top of another, would enable the development of materials with highly beneficial features. In particular, one could formulate completely new materials where each layer encompasses a distinct property and thus, the composite could exhibit a range of novel behavior [1]. In other words, one could compartmentalize different functionalities into the different layers and incorporate a new functionality by simply adding a new layer. Additionally, the approach would allow one to repair the gel if layers are severed [2]. Notably, the new layer could be grown from reactive chain ends in the existing underlying layer. This process would ensure the formation of covalent bonds between the different layers, and thus, the creation of strong interfaces between the different gels.

Of particular interest is creating stacked gels where one layer is hydrophilic and the other layer is hydrophobic [3]. Such tailored amphiphilic gel systems would be particularly effective for the staged release of different encapsulated species. Namely, the presence of both hydrophobic and hydrophilic domains enables the system to carry oil-based and waterbased components. The latter characteristics are especially important for controlled drug release applications [4,5]. Formulated as a thin film, the multi-stack gels could be applied as 
a dermal patch, releasing drugs through the skin. Importantly, the number of layers and polymer density in each layer could be tailored to optimize the desired rate of release.

Using both computer simulations and experiments, we previously demonstrated [6] the successful formation of multi-layered, stackable gels through the use of living copolymerization. Specifically, we placed a solution of initiator, monomer and cross-linker on top of an already polymerized gel in the same solvent; this new solution underwent living copolymerization to form a second gel on top of the first layer. Using dissipative particle dynamics (DPD) simulations, we developed schemes to model the growth of polymer chains in two distinctly different modes: (i) chain growth mimicking free radical polymerization (FRP) [7-9], in which the chains are slowly initiated and terminated and (ii) living chain growth, such as atom transfer radical polymerization (ATRP) [10-13], in which essentially chains are rapidly initiated, propagate concurrently and retain the ability to extend throughout the entire course of polymerization. Through this approach, we showed that reactive chain ends in the first gel, and not the partially reacted cross-linkers, were primarily responsible for the formation of covalent bonds between the gel layers. The complementary experiments demonstrated that for compatible gel layers in the same solvent, the resulting material was mechanically robust when the gels were formed either by ATRP or FRP. These experiments corroborated the simulation results that strong inter-gel bonds were formed between the layers. In the case of incompatible gel layers in the same solvent, the experimental studies showed that multi-layered gels formed via ATRP were mechanically more robust than stacked layers formed via FRP. In particular, the gels formed using FRP broke into separate pieces when the material was bent. In an attempt to bind incompatible gel layers, we introduced amphiphilic miktoarm star polymers during the fabrication of multilayer stackable gels, which were formed by sequential controlled radical polymerization of successive layers in incompatible solvents [14]. Both the simulations and experiments revealed that systems 
fabricated via ATRP formed mechanically interconnected bilayer stacks. Notably, without the addition of the miktoarm stars, the gels did not form connected layers. The DPD simulations showed that the miktoarm stars localized at the interface and acted as a compatibilizer, forming a bridge between the gel layers.

In the above computational study, the monomers for the different gels were taken to be highly incompatible to match the experimental systems under consideration [14]. The two solvents were also taken to be mutually incompatible. (The monomers were, however, compatible with their respective solvents.) Given the repulsive interactions in this system and the absence of active radicals in the lower gel, the compatibilizer was vital to the mechanical integrity of the stacked gel. Herein, we retain the incompatible solvents, but now consider the experimentally realizable system where the monomers are only weakly incompatible. The latter system lies between two extreme cases, where all the components are mutually compatible [6] or all the components are immiscible [14].

Given the decreased repulsion between the monomers in the above system, our aim in the present study is to determine via the DPD simulations if ATRP or FRP are sufficient to form a robust two-layer stack of gels without the addition of compatibilizer. We consider all possible combinations of these living radical polymerization approaches for forming the twolayered gel: ATRP-ATRP, ATRP-FRP, FRP-ATRP, and FRP-FRP. Moreover, by focusing on the four different scenarios, we can gain deep insight into the nature of the interface formed between the gels when the different polymerization techniques are applied to the first and the subsequent layers. Since the DPD simulations capture phenomena up to $100 \mathrm{~nm}$ in length scale, these computer simulations allow us to zoom in the nanoscopic features of the critical interfacial region.

Below, we begin by describing the salient features of DPD [15-18], which can be viewed as a coarse-grained molecular dynamics (MD) approach. For instance, a DPD bead 
represents clusters of molecules rather than a single atom, as is typically used in MD simulations. By setting the values of the interaction parameters between the beads (Table 1), we can specify the degree of compatibility between all the different components. In the subsequent section, we discuss our findings on the factors that control the inter-gel bonding between the two layers, and thereby dictate the structural integrity of the materials.

\section{Methodology}

We use dissipative particle dynamics (DPD) [15-18] to model the dynamic behavior and radical polymerization processes occurring within this multi-component system. DPD is a particle-based approach used to simulate the time evolution of a many-body system governed by Newton's equation of motion: $m d \mathbf{v}_{i} / d t=\mathbf{f}_{i}$. The force $\mathbf{f}_{i}(t)=\sum\left(\mathbf{F}_{i j}^{C}+\mathbf{F}_{i j}^{D}+\mathbf{F}_{i j}^{R}\right)$ acting on a bead $i$ is the sum of three pairwise additive forces. The sum runs over all beads $j$ within a certain cutoff radius $r_{c}$ from bead ${ }_{i}$. The three pairwise forces are the conservative force $\mathbf{F}_{i j}^{C}$, drag or dissipative force $\mathbf{F}_{i j}^{D}$, and random force $\mathbf{F}_{i j}^{R}$; each of these are described below.

The conservative force $\mathbf{F}_{i j}^{C}=a_{i j}\left(1-r_{i j}\right) \hat{\mathbf{r}}_{i j}$ is a soft repulsive force acting between beads $i$ and $j$ that are separated by a distance $r_{i j}=\left|\mathrm{r}_{i}-\mathrm{r}_{j}\right| / r_{c}$; the force is linear up to the cutoff distance $r_{c}$. Here $a_{i j}$ measures the maximum repulsion between the beads (see Table 1), and $\hat{\mathbf{r}}_{i j}=\mathbf{r}_{i j} /\left|\mathbf{r}_{i j}\right|$ describes the direction of the force. This soft-core force leads to a degree of overlap between neighboring beads and permits the use of larger time steps than those typically used in MD simulations, which commonly involve hard-core potentials (e.g., the Lennard-Jones potential) [17].

The dissipative force $\mathbf{F}_{i j}^{D}=-\gamma \omega_{D}\left(r_{i j}\right)\left(\hat{\mathbf{r}}_{i j} \cdot \mathbf{v}_{i j}\right) \hat{\mathbf{r}}_{i j}$ between two beads is linear in the relative momentum $\mathbf{v}_{i j}=\mathbf{v}_{i}-\mathbf{v}_{j}$, where $\gamma$ is a simulation parameter related to the viscosity 
arising from the interactions between beads (friction coefficient), $\omega_{D}$ is a weight function that goes to zero at $r_{c}$. The random force is $\mathbf{F}_{i j}^{R}=\sigma \omega_{R}\left(r_{i j}\right) \xi_{i j} \hat{\mathbf{r}}_{i j}$, where $\xi_{i j}$ represents a zero-mean Gaussian random variable of unit variance and $\sigma$ is the amplitude of the noise. The equality $\sigma^{2}=2 \gamma K_{B} T$ relates the noise amplitude to the friction coefficient, as specified by the fluctuation-dissipation theorem [16]. The value of $\gamma=4.5$ is chosen to ensure relatively rapid equilibration of the temperature of the system and the numerical stability of the simulations for the specified time-step [17]. Here, $k_{B}$ is the Boltzmann constant, and $T$ is the temperature of the system. We select the weight functions to take the following form: $\omega_{D}\left(r_{i j}\right)=\omega_{R}\left(r_{i j}\right)^{2}=\left(1-r_{i j}\right)^{2}$ for $r_{i j}<r_{c}$ [17]. Each of these three pairwise forces conserves momentum locally, and thus, DPD reproduces the correct hydrodynamic behavior even in systems containing only a few hundred particles $[16,17]$.

We integrate the equation of motion by use of the modified velocity Verlet algorithm [19]. In our simulation, we take $r_{c}$ as the characteristic length scale and set the dimensionless value as $r_{c}=1$. The factor $k_{B} T$ is taken as the characteristic energy scale. We chose room temperature as the reference value; thus, we set $k_{B} T_{0}=1$ with $T_{0}=298.15^{\circ} \mathrm{K}$. The interaction parameter $a_{i j}$ is given in terms of $k_{B} T_{0} / r_{c}$; the reduced temperature is then introduced as $T^{*}=T / T_{0}$, and the characteristic time scale is defined as $\tau=\left(m r_{c}^{2} / k_{B} T_{0}\right)^{1 / 2}$. The remaining simulation parameters [2] are specified by setting the simulation time step to $\Delta t=0.02 \tau$, and the total bead number density equal to $\rho=3$.

The polymer chains are simulated as a bead-spring model with a harmonic bond potential between neighboring DPD beads that is given by $E_{b}=1 / 2 K_{b}\left(r-r_{0}\right)^{2}$. Here, $K_{b}=128$ is the elastic constant and $r_{0}=0.5$ is the equilibrium bond distance. 
To simulate the formation of polymer gel layers, we utilize our recently developed DPD approach for living radical polymerization. Namely, we model the ATRP $[6,20,21]$ and FRP $[6,22]$ processes by a particular set of appropriate elemental reactions. The reaction kinetics is coupled to the dynamics of the system, which is evolved by the DPD simulations. The reactive species in the system are the initiator (I), bifunctional cross-linker (X), and monomer (M) which all are modeled as DPD beads. The bifunctional cross-linker encompasses two reactive cross-linking units and is modeled by one DPD bead with four different "states", which indicate the effective reactivity of the cross-linker (i.e., the extent to which it has reacted) $[6,20,21]$. These "states" are as follows: single-bonded state where one cross-linking unit is in the active state and the other unit is unreacted; double-bonded state where one unit is fully reacted and the other is unreacted; triple-bonded state where one unit is fully reacted and the other unit is activated and finally, the fully reacted state where both crosslinking units are fully reacted, resulting in the formation of four bonds. Since the probability of forming the doubly active state where both cross-linking units are active is very low; we neglect this state in our simulation.

Due to the characteristics of living polymerization processes, we exclude termination reactions in ATRP [10-13]. Chain termination, however, is prominent in FRP, and thus, the termination reactions between active chain ends are considered in our simulations of FRP. In particular, we focus on radical combination and disproportionation as the two mechanisms of chain termination in FRP [23]. Chain transfer reactions are excluded in the simulation [24].

The polymerization process in our approach is similar to that previously modeled via coarse-grained MD and MC simulations [23,25-30]. In each reaction step, we select an active radical and for that selected radical, we randomly choose another bead within the interaction radius $r_{i}$. The selected pair of beads forms a bond with a polymerization probability $P_{r}^{x}$ [26]. Here, the superscript $x$ stands for the type of reaction, which depends upon the type of 
selected beads. Each successful reaction results in irreversible bond formation. In our simulation, we choose different probabilities for the reactions: the initiation probability $P_{r}^{i}$, the monomer addition (propagation) probability $P_{r}^{p, M}$, and the cross-linking probability $P_{r}^{p, X}=2 P_{r}^{p, M}$. The cross-linking probability of the pendent group is selected as $P_{r}^{p, P}=3 P_{r}^{p, M}$ to account for the fact that a single bead models the bifunctional cross-linker [26]. (A "pendent group" refers to a partially reacted cross-linker that is linked to at least two polymerized chains.) The termination (through combination and disproportionation) probabilities are represented by $P_{r}^{t, C}$ and $P_{r}^{t, D}$, respectively. The schematics of the bead types and the major reactions (for both ATRP and FRP processes) are summarized in Fig. 1 and the probability values are given in Table 2 .

We can effectively adjust the rate constants of the respective reactions by choosing different reaction probabilities [6,20-22]. The value of probabilities should, however, be sufficiently small to ensure controlled polymerization growth in the kinetically controlled reaction regime [30]. We set the interaction radius to be $r_{i}=0.7$ since smaller values of $r_{i}$ result in delayed gelation and taking $r_{i}>0.7$ led to considerable deviations from linear firstorder kinetics $[20,26]$. The reactions are performed every ten simulation time steps [27].

We first model the bulk polymerization in a 3D system with either the ATRP or FRP processes. The size of our simulation box is $25 \times 25 \times(25 N)$, where $N$ stands for the number of layers. Periodic boundary conditions are imposed in the lateral ( $x$ and $y$ ) directions. The solid walls bound the system in the transverse $(z)$ direction; the top and bottom walls are modeled by repulsive beads (with a height $h=1$ and density $\rho_{\text {wall }}=3$ ) that effectively repel the gel and all the rest of the beads in the system [31]. Bounce-back boundary conditions are applied at the fluid-solid interfaces to prevent solvent and gel beads from penetrating into the 
walls, and produce no-slip boundary conditions with minimal interfacial density oscillations [18].

We generate the initial configuration of each new solution, which contains initiator, monomer, cross-linker, and solvent $(\mathrm{S})$, by randomly placing these different beads in a box of size $25 \times 25 \times 25$. The ratio of the initial concentration of these respective species is $[\mathrm{I}]_{0} /[\mathrm{X}]_{0} /[\mathrm{M}]_{0}=1 / 5 / 75$, and the corresponding solvent concentration is $[\mathrm{S}]_{0}=50 \%$. The total bead number density of the system is $\rho=3$. We equilibrate each solution for $5 \times 10^{4}$ simulation time steps before allowing the polymerization reaction to proceed. This process ensures that the diffusion between the current gel layer and the new solution is not influenced by the initial configuration of the solution.

The interaction parameter between any two beads that are compatible is set to $a_{i j}=25$ (in units of $k_{B} T / r_{c}$ ), while the interaction parameter between incompatible species is set to $a_{i j}=60$ (see Table 1 ). To model the fact that the two gels are slightly incompatible, we set the interaction parameter between two monomers within the different gels to $a_{i j}=35$.

\section{Simulation Results}

\section{A. Single Gel Layer}

We perform simulations to examine the properties of two-layered gels, referring to the monomers that form the lower gel as M1 and monomers that form the upper gel as M2. The solvent for M1 is designated as $\mathrm{S} 1$ and the solvent for M2 is referred to as $\mathrm{S} 2$. As indicated in Table 1, the monomers are compatible with their respective solvents; additionally, M1 is incompatible with S2, but M2 is compatible with S1. For example, we assume that S1 represents toluene and S2 is water; M1 can represent butyl methacrylate and M2 can represent 2-(dimethylamino)ethyl methacrylate [2]. As can be realized experimentally [2], the 
cross-linkers were taken to be compatible with both solvents, as well as all the other components, i.e., $a_{i j}=25$ (Table 1 ).

Since the gels are grown via ATRP or FRP, it is important that our simulations accurately capture the appropriate polymerization kinetics that characterizes these different processes. Hence, we first focus on a single gel layer to analyze the kinetics of polymerization via both ATRP and FRP. The time evolution of the monomer conversion for both ATRP and FRP is plotted in Fig. 2a; the plots reveal that our choices for the reaction probabilities (i.e., kinetics and the overall rate of polymerization) lead to $\sim 95 \%$ monomer conversion at approximately the same time for both processes. Thus, gels grown by either ATRP or FRP will take the same time to reach $95 \%$ monomer conversion. In Fig. 2 b, the plot of $\ln \left([\mathrm{M}]_{0} /[\mathrm{M}]\right)$ as a function of time shows linear, first-order kinetics for the ATRP process since the concentration of active chain ends remains constant. In FRP, the system deviates from first-order kinetics because the rates of initiation and termination are similar in this process (leading to a steady-state concentration of radicals).

ATRP is characterized by a fast initiation reaction. The blue line in Fig. 2c shows that our choice of probabilities (Table 2) for the ATRP process correctly captures the fast initiation for the ATRP gel. Since ATRP is essentially a living copolymerization process, termination is neglected and all the initiated chains propagate as long as monomers are available, i.e., the total concentration of active chain ends (monomer-capped active chain ends and active chain ends bound to cross-linkers) is equal to the concentration (number) of activated initiators. (In Fig. 2c, the red line shows only the number of monomer-capped active chain ends and hence, is not equal to the total number of initiators.) On the other hand, FRP is characterized by slow initiation and fast termination; as shown in Fig. 2d, our simulations capture this salient feature of free radical polymerization. 
We can gain further insight into the differences between these two polymerization processes by characterizing the morphologies of gels grown via ATRP versus FRP. It is, however, useful to first consider the behavior of these processes in the absence of crosslinkers. Since all the initiators undergo fast activation and there is no termination, all the chains propagate at the same rate and the ATRP polymer chains display low dispersity (see the inset in Fig. 3a). For FRP, the slow initiation and fast propagation kinetics leads to the formation of long chains. Termination by combination leads to longer polymer chains, while termination by disproportionation leads to shorter chains. The inset in Fig. 3 b shows that chain length is spread over a large range ( $\sim 3000$ beads), emphasizing the polydisperse nature of FRP chains. The presence of cross-linker restricts the FRP chains from growing to such long lengths; however, the maximum chain length (i.e., beads between cross-links) is three times that of ATRP chains. The dispersity in chain lengths for the FRP versus the ATRP gel is reflected in the images in Figs 3a-b.

\section{B. Two-layer Gel Systems}

We carried out simulations for the four possible means of forming the two-layered gel: ATRP-ATRP, ATRP-FRP, FRP-ATRP, and FRP-FRP. Unless mentioned otherwise, the abbreviation on the left denotes the polymerization process carried out in the lower gel and the abbreviation on the right one denotes the polymerization process in the upper gel. For example, ATRP-FRP indicates that the lower gel was grown via ATRP and the upper gel was grown via FRP.

In our simulations, we assume that different initiators are used for the two different gel layers, i.e., the initiation rates are different for the two polymerization processes. As noted above, the monomers in the lower gel (M1) are incompatible with the solvent in upper layer; however, the monomers in the upper gel (M2) are compatible with the lower solvent and the 
cross-linkers are compatible with both solvents and gel monomers. Hence, free cross-linkers and free M2 monomers can diffuse into the lower gel layer.

Below, we separate the discussion into two sections; we first describe scenarios where the lower gel is formed by ATRP and hence, analyze the ATRP-ATRP and ATRP-FRP systems. We then discuss the cases where the lower gel is formed by FRP: FRP-ATRP and FRP-FRP. In the following section, I1 and X1 denote the respective initiator and cross-linker in the bottom gel (G1). Similarly, I2 and X2 denote the respective initiator and cross-linker in the top gel (G2).

\section{Lower Gel formed by ATRP}

We follow a two-step polymerization process to form these two-layered gels. In the first step, we create the lower ATRP gel by introducing the following ratio of initiator, monomer, and cross-linker concentration into the solvent $\mathrm{S} 1:[\mathrm{I} 1]_{0} /[\mathrm{X} 1]_{0} /[\mathrm{M} 1]_{0}=1 / 5 / 75$. The gel undergoes living polymerization and the simulation follows the methodology for creating an ATRP gel discussed above. Once the monomer conversion reaches 95\%, further polymerization is not continued. All the chain ends (monomer-capped chain ends and active cross-linkers) are active and participate in further reactions when the second step takes place, i.e., the polymerization of the upper gel layer. The remaining free, unreacted monomers M1 of the lower gel G1, however, do not react during the successive polymerization step. The lower ATRP gel is shown in blue in Figs. 4a-b.

We then double the height of the simulation box and move the bounding wall to the new top layer. Into the upper half of this new simulation box, we introduce the immiscible solvent S2 on top of the first gel layer (G1) and add the second initiator, cross-linker and monomer at following ratios: $[\mathrm{I} 2]_{0} /[\mathrm{X} 2]_{0} /[\mathrm{M} 2]_{0}=1 / 5 / 75$. The $\mathrm{I} 2$ initiators are incompatible with S1 and hence, cannot diffuse into the lower gel layer containing solvent S1. 
The M2 monomers can diffuse into solvent S1 and the active ends of the lower gel layer can participate in reactions. Hence, there exists a finite probability of a reaction between M2 and moieties in G1. Specifically, the reactive chain ends, and the partially reacted cross-linkers in the lower gel can react with the monomers and cross-linkers of the upper gel layer. These are the cross-reactions that can take place between the two gel layers. The latter cross-reaction probability was kept the same as the relevant probabilities for the lower gel because both the lower and upper gels follow the ATRP polymerization mechanism. Since the initiation of ATRP in the upper gel and the cross-propagation probability of reactive species in G1 are the same, polymerization of G2 also starts in the lower portion of the gel and these chains bridge the lower and upper gel. The upper ATRP gel layer is shown in green in Fig. 4a. The patches of green inside the blue gel indicate the polymerization of free M2 monomers in the lower region. The interface between the blue and green gels shows a roughness that indicates the formation of covalent bonds between these two layers. It should be emphasized that the monomer in upper gel is compatible with the lower solvent and hence cross-linkers and monomers can diffuse into the lower gel layer.

For the ATRP-ATRP system, approximately 100 inter-gel covalent bonds are formed at the interface between G1 and G2 (red curve in Fig. 5a). Since M2 can diffuse into S1 and the active radicals present in M1 have the same probability of propagation as the propagation probability in the upper layer, patches of G2 grow in the lower gel and are not long enough to reach the interface (see Fig. 4a, patches of green gel inside blue gel). The plot of inter-gel bond formation in Fig. $5 \mathrm{~b}$ for the entire gel also shows increased number of bonds.

For the ATRP-FRP gel, the cross-reaction probability was chosen to be lower than the ATRP propagation probability. Once the cross-reactions are initiated, further propagation into the lower gel proceeds with FRP propagation probabilities. This leads to the formation of inter-gel bonds, but the number of inter-gel covalent bonds formed at the interface (blue 
curve in Fig 5a) is significantly less than those in the ATRP-ATRP case. Figure 5b also shows that the total inter-gel bonds formed in ATRP-FRP is lower than the number in the ATRP-ATRP gel.

Notably, the density of the gel along the $z$ direction is continuous for the lower and upper gel regions (Fig. 5c); however, there is a dip at the interface between G1 and G2, indicating that there are fewer covalent bonds in the interfacial region than the rest of the gel. The dip in the density is due to the fact that the solvents are immiscible and the monomers forming the respective gels are also incompatible. The dip, however, is less pronounced for the ATRP-ATRP gel than the ATRP-FRP gel due to the greater number of bonds that are formed in the ATRP-ATRP case.

\section{Lower Gel formed by FRP}

We now consider the behavior of systems where the bottom gel is formed from FRP. As we show below, the two-layered gel FRP-ATRP and FRP-FRP systems (Figs. 4c-d) display behavior that is in sharp contrast to the ATRP-ATRP and ATRP-FRP gels. Namely, when FRP is used to create the lower layer, a negligible number of inter-gel covalent bonds are formed across the interface.

As mentioned above, we utilize a two-step polymerization process to create the twolayered gel. In the first step, the lower FRP gel is prepared by introducing the initiator, crosslinker and monomer in the S1 solvent at the following respective ratios: $[\mathrm{I} 1]_{0} /[\mathrm{X} 1]_{0} /[\mathrm{M} 1]_{0}=$ 1/5/75. Unlike the ATRP gel, where all monomer-capped chain ends remain active, most chain ends are terminated at 95\% monomer conversion in FRP gel due to the termination step in the FRP process (see Table 2). (In our simulations, there are less than six active chain ends for this scenario.) 
During the polymerization of the upper gel layer, only cross-linkers of the lower FRP gel participate in the cross-reactions. The free beads (I1/X1/M1) and the few non-terminated chain ends $(\mathrm{M} 1 *)$ do not undergo further reactions during the successive polymerization step.

Given the presence of cross-linkers, both ATRP and FRP polymerization processes lead to the formation of a gel in the upper layer. As noted above, the gel monomers are mutually incompatible, as are the solvents in both layers. Figures $4 \mathrm{c}$ and $4 \mathrm{~d}$ show that the mutually repulsive gel layers form distinct layers that are separated by a sharp interface. This observation is substantiated in Fig $5 \mathrm{~d}$ where the temporal evolution of the number of inter-gel bonds $\left(\mathrm{N}_{\mathrm{ICB}}\right)$ near the interface shows that throughout the polymerization of the upper layer, for both FRP-ATRP (in green) and FRP-FRP (in violet), the value of $\mathrm{N}_{\mathrm{ICB}}$ is negligible (less than five in our simulations).

Recall that M2 and X2 are compatible with $\mathrm{S} 1$; hence, the free monomers and crosslinkers of G2 can diffuse into the lower gel region. We do not, however, observe large patches of G2 inside G1, suggesting that the number of cross-reactions between active ends of G1 and freely diffusing (green) monomers and cross-linkers is low for both the FRPATRP and FRP-FRP gel. Figure 5e shows the total number of inter-gel bonds $\mathrm{N}_{\mathrm{TCB}}$ formed in the entire two-layered gel system. The value of $\mathrm{N}_{\mathrm{TCB}}$ is smaller for the FRP-ATRP (in green) than the FRP-FRP (in violet) gel. These numbers reflect the fact that the patch of new (green) gel inside blue is smaller for the FRP-ATRP (Fig. 4c) gel than FRP-FRP sample (Fig. 4d).

Plots of the number density of the two-layered gel (i.e. all the gel beads belonging to the gel) in Fig. 5f shows that the density drops at the G1-G2 interface, indicating a weak connection between the lower and upper gel, as well as the effective repulsion between the gels. Since the initiation reaction could not take place in the lower gel, new chains inside the lower gel region are only possible due to the cross-reaction between the active cross-linker of lower gel and freely diffusing M2 monomers or X2 cross-linkers of the upper gel layer. 
(Recall that initiation reactions are terminated once we introduce a solution in the top layer.) Once a cross-reaction is triggered, further reaction proceeds via the respective polymerization probability of the upper gel layer. The formation of small patches inside the lower gel and the sharp interface between the gel layers suggest that the cross-reaction required to form intergel covalent bonds did not take place to a significant degree.

Notably, for both cases involving a lower FRP gel layer, there were few inter-gel connections between the layers. We assumed that all radicals with monomer-capped active chain ends are terminated in the bottom gel (G1) grown via FRP. Thus, one obvious reason for the small amount of new gel growing inside the already grown lower gel is the lack of monomer-capped chain ends.

Furthermore, it is useful to consider the behavior of the cross-linkers since only crosslinkers with active chain ends can produce cross reactions in the gel. As mentioned above, the doubly functional cross-linker can be present in any one of the four "states". By examining the number of cross-linkers in these different states, we found that most of the cross-linkers that could participate in the second stage polymerization above a G1 FRP gel are in the double-bonded state. Only a few (less than four) were in the single- or triple-bonded state. In the case involving a G1 gel grown via ATRP, the number of single-, double-, triple-bonded cross-linkers were high (greater than 30) in our simulation. These cross-linkers are still not fully reacted, and they are not in the active state [26]; therefore, active chain ends are required to react with these cross-linkers in order for polymerization to occur. The dominance of cross-linkers in the double-bonded state could be due to the higher value of the chain termination probability in FRP that leads to fast termination of monomer-capped active chain ends. A cross-linker in the double-bonded state is bound to the gel; this restricts the mobility of these cross-linkers and hence, limits access to active radicals. Thus, on growing the top gel via ATRP or FRP, the monomers and cross-linkers diffusing into the bottom gel layer have 
few active chain ends to interact with and form cross bonds; this results in very few cross bonds for the FRP-ATRP and FRP-FRP gel.

\section{Conclusions}

Using DPD simulations that incorporate reaction schemes for the formation of gels through ATRP and FRP, we investigated the ability to create mechanically robust materials that encompass incompatible gels and solvents. We first characterized the features of a single gel layer formed via ATRP or FRP. The simulation results accurately captured the kinetics and overall rate of polymerization for the two different polymerization processes.

We then modeled two-layer gel systems that are formed through the following sequential processes: ATRP-ATRP, ATRP-FRP, FRP-ATRP, and FRP-FRP. When the lower gel layer is formed through ATRP, a significant number of inter-gel bonds are formed at the interface between the two layers. On the other hand, when the lower gel is formed with FRP, essentially no inter-gel bonds are formed, regardless whether the subsequent layer is formed with ATRP or FRP. Notably, the presence of active radicals in the lower gel (before the polymerization of the solution to form the upper layer) is an important factor that contributes to the inter-gel bond formation. The ATRP gels encompass active radicals since the process does not involve termination reactions. In the case of the FRP gels, however, all the monomer-capped chain ends are terminated; although cross-linkers are present in the system, most of these species require active radicals to form bonds.

In summary, for two incompatible gels that are brought together through a combination of ATRP and FRP, the vital inter-gel bonds are formed only when the lower gel is created via ATRP. Reversing the order of the processes (i.e., forming the layers via FRPATRP) does not lead to an appreciable number of inter-gel bonds between the two layers, and thus, could lead to material that is not mechanically robust. The active radicals in the lower ATRP gel results in the formation of covalent bonds between gel layers, and thus yields an 
amphiphilic gel with mechanical integrity. The lack of active radicals in the lower FRP gels leads to a sharper interface with negligible inter-gel contacts. The findings from these studies can provide valuable guidelines for creating multifunctional "stacked" gels in incompatible solvent without the need of adding compatibilizers (such as amphiphilic miktoarm stars), potentially making the fabrication of these useful materials more cost-effective.

\section{Acknowledgments}

Financial support from Department of Energy (grant ER45998) is gratefully acknowledged. 


\section{References}

[1] S.J. Banik, N.J. Fernandes, P.C. Thomas, S.R. Raghavan, A New Approach for Creating Polymer Hydrogels with Regions of Distinct Chemical, Mechanical, and Optical Properties, Macromolecules. 45 (2012) 5712-5717. doi:10.1021/ma300859b.

[2] E.B. Stukalin, L.-H. Cai, N.A. Kumar, L. Leibler, M. Rubinstein, Self-Healing of Unentangled Polymer Networks with Reversible Bonds, Macromolecules. 46 (2013) 7525-7541. doi:10.1021/ma401111n.

[3] G. Deng, Q. Ma, H. Yu, Y. Zhang, Z. Yan, F. Liu, C. Liu, H. Jiang, Y. Chen, Macroscopic Organohydrogel Hybrid from Rapid Adhesion between Dynamic Covalent Hydrogel and Organogel, ACS Macro Lett. 4 (2015) 467-471. doi:10.1021/acsmacrolett.5b00096.

[4] V. Prasad, N. Kumar, P.R. Mishra, Amphiphilic Gels as a Potential Carrier for Topical Drug Delivery, Drug Deliv. 14 (2007) 75-85. doi:10.1080/10717540600642431.

[5] E. Caló, V. V. Khutoryanskiy, Biomedical applications of hydrogels: A review of patents and commercial products, Eur. Polym. J. 65 (2015) 252-267. doi:10.1016/j.eurpolymj.2014.11.024.

[6] X. Yong, A. Simakova, S. Averick, J. Gutierrez, O. Kuksenok, A.C. Balazs, K. Matyjaszewski, Stackable, Covalently Fused Gels: Repair and Composite Formation, Macromolecules. 48 (2015) 1169-1178. doi:10.1021/ma502367f.

[7] K. Matyjaszewski, T.P. Davis, eds., Handbook of Radical Polymerization, John Wiley \& Sons, Inc., Hoboken, NJ, USA, 2002. doi:10.1002/0471220450.

[8] G. Moad, D.H. Solomon, The Chemistry of Radical Polymerization, Elsevier, 2005.

[9] F. Di Lorenzo, S. Seiffert, Nanostructural heterogeneity in polymer networks and gels, Polym. Chem. 6 (2015) 5515-5528. doi:10.1039/C4PY01677G.

[10] J.S. Wang, K. Matyjaszewski, Controlled Living Radical Polymerization - Atom- 
Transfer Radical Polymerization in the Presence of Transition-Metal Complexes, J. Am. Chem. Soc. 117 (1995) 5614-5615. doi:Doi 10.1021/Ja00125a035.

[11] K. Matyjaszewski, J.H. Xia, Atom transfer radical polymerization, Chem. Rev. 101 (2001) 2921-2990. doi:10.1021/cr940534g.

[12] K. Matyjaszewski, Atom Transfer Radical Polymerization (ATRP): Current Status and Future Perspectives, Macromolecules. 45 (2012) 4015-4039. doi:10.1021/ma3001719.

[13] K. Matyjaszewski, N. V Tsarevsky, Macromolecular Engineering by Atom Transfer Radical Polymerization, J. Am. Chem. Soc. 136 (2014) 6513-6533. doi:10.1021/ja408069v.

[14] A. Beziau, A. Singh, R.N.L. de Menezes, H. Ding, A. Simakova, O. Kuksenok, A. Balazs, T. Kowalewski, K. Matyjaszewski, Miktoarm star copolymers as interfacial connectors for stackable amphiphilic gels, Polymer (Guildf). (2016). doi:10.1016/j.polymer.2016.08.070.

[15] P.J. Hoogerbrugge, J.M.V.A. Koelman, Simulating Microscopic Hydrodynamic Phenomena with Dissipative Particle Dynamics, Europhys. Lett. 19 (1992) 155-160. doi:Doi 10.1209/0295-5075/19/3/001.

[16] P. Espanol, P. Warren, Statistical-Mechanics of Dissipative Particle Dynamics, Europhys. Lett. 30 (1995) 191-196. doi:Doi 10.1209/0295-5075/30/4/001.

[17] R.D. Groot, P.B. Warren, Dissipative particle dynamics: Bridging the gap between atomistic and mesoscopic simulation, J. Chem. Phys. 107 (1997) 4423-4435. doi:Doi 10.1063/1.474784.

[18] D.A. Fedosov, I. V Pivkin, G.E. Karniadakis, Velocity limit in DPD simulations of wall-bounded flows, J. Comput. Phys. 227 (2008) 2540-2559. doi:10.1016/j.jcp.2007.11.009.

[19] S. Plimpton, Fast Parallel Algorithms for Short-Range Molecular-Dynamics, J. 
Comput. Phys. 117 (1995) 1-19. doi:DOI 10.1006/jcph.1995.1039.

[20] X. Yong, O. Kuksenok, K. Matyjaszewski, A.C. Balazs, Harnessing interfaciallyactive nanorods to regenerate severed polymer gels, Nano Lett. 13 (2013) 6269-6274.

[21] A. Singh, O. Kuksenok, J.A. Johnson, A.C. Balazs, Tailoring the structure of polymer networks with iniferter-mediated photo-growth, Polym. Chem. 7 (2016) 2955-2964. doi:10.1039/C6PY00325G.

[22] X. Yong, O. Kuksenok, A.C. Balazs, Modeling free radical polymerization using dissipative particle dynamics, Polymer (Guildf). 72 (2015) 217-225. doi:10.1016/j.polymer.2015.01.052.

[23] J. Genzer, In silico polymerization: Computer simulation of controlled radical polymerization in bulk and on flat surfaces, Macromolecules. 39 (2006) 7157-7169. doi:Artn Ma061155f10.1021/Ma061155f.

[24] A. Giz, H. Çatalgil-Giz, A. Alb, J.-L. Brousseau, W.F. Reed, Kinetics and Mechanisms of Acrylamide Polymerization from Absolute, Online Monitoring of Polymerization Reaction, Macromolecules. 34 (2001) 1180-1191. doi:10.1021/ma000815s.

[25] R.L.C. Akkermans, S. Toxvaerd, W.J. Briels, Molecular dynamics of polymer growth, J. Chem. Phys. 109 (1998) 2929-2940. doi:Doi 10.1063/1.476845.

[26] H. Gao, P. Polanowski, K. Matyjaszewski, Gelation in living copolymerization of monomer and divinyl cross-linker: Comparison of ATRP experiments with Monte Carlo simulations, Macromolecules. 42 (2009) 5925-5932. doi:10.1021/ma901005d.

[27] H. Liu, M. Li, Z.Y. Lu, Z.G. Zhang, C.C. Sun, Influence of Surface-Initiated Polymerization Rate and Initiator Density on the Properties of Polymer Brushes, Macromolecules. 42 (2009) 2863-2872. doi:10.1021/ma802817r.

[28] A. Milchev, J.P. Wittmer, D.P. Landau, Formation and equilibrium properties of living 
polymer brushes, J. Chem. Phys. 112 (2000) 1606-1615. doi:Doi 10.1063/1.480600.

[29] P. Polanowski, J.K. Jeszka, K. Matyjaszewski, Modeling of branching and gelation in living copolymerization of monomer and divinyl cross-linker using dynamic lattice liquid model (DLL) and Flory-Stockmayer model, Polymer (Guildf). 51 (2010) 60846092. doi:10.1016/j.polymer.2010.10.006.

[30] S. Turgman-Cohen, J. Genzer, Computer Simulation of Controlled Radical Polymerization Effect of Chain Confinement Due to Initiator Grafting Density and Solvent Quality in “Grafting From” Method, Macromolecules. 43 (2010) 9567-9577. doi:10.1021/ma102046q.

[31] A.C.C. Esteves, K. Lyakhova, L.G.J. van der Ven, R.A.T.M. van Benthem, G. de With, Surface Segregation of Low Surface Energy Polymeric Dangling Chains in a Cross-Linked Polymer Network Investigated by a Combined Experimental-Simulation Approach, Macromolecules. 46 (2013) 1993-2002. doi:10.1021/ma302236w. 


\section{Figure Captions}

Figure 1: Schematic of all the reactions in the radical polymerization considered in the simulation. Note that termination is only considered for the FRP process. Details of the living copolymerization steps are given in Gao et al.[26], and details of the simulation in the DPD framework is provided in Yong et al. [20,22]. Also, the doubly active bifunctional crosslinking state is ignored in our simulation.

Figure 2: (a) Conversion of monomer and cross-linker during the polymerization for gels grown via ATRP and FRP, where $[\mathrm{M}]$ or $[\mathrm{X}]$ is the current concentration of reacted monomer or cross-linker, respectively. For both ATRP and FRP, the conversion of monomer and crosslinker is attained at the same time. (b) $\ln \left([\mathrm{C}]_{0} /[\mathrm{C}]\right)$ as a function of simulation time steps, where $C=\{M, X\}$ and $[C]$ denotes current concentration of the unreacted monomer $[\mathrm{M}]$ or cross-linker $[\mathrm{X}]$. (c) In ATRP, fast initiation (blue line) and large number of monomercapped chain ends show living copolymerization whereas in (d), the FRP gel shows only a few initiators reacted during the polymerization process i.e., slow initiation (blue line) and almost all chain ends are terminated, fast termination (red line).

Figure 3: The probability density function of the chain length for (a) ATRP (b) FRP. (a) Shows the monodisperse nature of ATRP. The inset shows the PDF for chains without crosslinkers. (b) Polydisperse nature of FRP. The inset shows the PDF for chains without crosslinkers.

Figure 4: Two-layered gel grown by the processes of (a) ATRP - ATRP (b) ATRP - FRP (c) FRP - ATRP and (d) FRP - FRP. Gel1 (blue) in the lower region is first prepared and then polymerization of gel2 (green) on top of the blue gel takes place. Solvents are not shown. Cross-linkers in the lower gel are represented by orange beads and in the upper gel by yellow beads. The simulation snapshot shown is for the last simulation time step equal to 150000 . 
Figure 5: The interfacial region is 10 units in the positive and negative direction along the $z$ direction from the maximum along the z-direction of gel 1 in the simulation box. (a) The lower gel is grown via ATRP. The number of inter-gel bonds at the interface i.e., bonds formed between G1 and G2 at the interface are more for ATRP - ATRP process (red) and less for the ATRP - FRP case in blue. (b) The plots show total number of inter-gel bonds in the entire gel. The increase in number of bonds is due to small patches of grown G2 inside G1 in addition to the interface bonds shown in (a). Number density of the two-layered gel along the $z$-direction in (c) for ATRP - ATRP (red) and ATRP - FRP (blue). The initial (lower) gel is grown by FRP in (d-f). We notice in (d) that negligible inter-gel bonds are formed at the interface between the lower and upper gel for both FRP- ATRP and FRP- FRP. (e) The total inter-gel bonds are higher because as mentioned earlier, patches of upper gel grows inside the lower gel. (f) shows the number density of the two-layered gel along the $z$ direction for FRP - ATRP (green) and FRP - FRP (purple). 


\section{Table Captions}

Table 1: The interaction parameter $a_{i j}$ used in the DPD simulation for different components. I1 and M1 denote the initiator and monomer for lower gel G1 in the solvent S1. Similarly, I2 and M2 denote the initiator and monomer for upper gel G2 in the solvent S2.

Table 2: Values used for the probability of initiation $P_{r}^{i}$, propagation with monomer $P_{r}^{p, M}$, propagation with cross-linker $P_{r}^{p, X}$ and pendent group $P_{r}^{p, P}$. Additionally, for FRP scheme, termination of chain ends via combination and disproportionation is considered with the probability of termination $P_{r}^{t}$. Considering chain combination to be the dominant termination reaction[22], $P_{r}^{t, c} / P_{r}^{t, D} \approx 4.7$. 


\section{Figures}

Initiation, Propagation \& Crosslinking

$$
\begin{aligned}
& \mathrm{O}^{*}+\mathrm{O} \stackrel{P_{r}^{i}}{\longrightarrow} \mathrm{O}-\mathrm{O}^{*} \\
& \sim \mathrm{O}^{*}+\mathrm{O} \stackrel{P_{r}^{p, M}}{\longrightarrow} \sim \mathrm{O}^{*} \\
& \mathrm{O}^{*}+\mathrm{O} \stackrel{2 P_{r}^{i}}{\longrightarrow} \mathrm{O}-\mathrm{O}^{*} \\
& \sim \mathrm{O}^{*}+\mathrm{O} \stackrel{P_{r}^{p, X}}{\longrightarrow} \sim \mathrm{O}^{*} \\
& \mathrm{O}^{*}+3 \stackrel{P_{r}^{i}}{\longrightarrow} \mathrm{O}^{3} \\
& \mathrm{OO}^{*}+\mathcal{2} \stackrel{P_{r}^{p, P}}{\longrightarrow} \sim \mathrm{S}^{*} \\
& \sim \mathrm{O}^{*}+\mathrm{O} \stackrel{P_{r}^{p, M}}{\longrightarrow} \sim \mathrm{O}^{*} \\
& \mathrm{~S}^{*}+\mathrm{O} \stackrel{P_{r}^{p, M}}{\longrightarrow} \sim \mathrm{S}^{*} \\
& \sim \mathrm{O}^{*}+\mathrm{O} \stackrel{P_{r}^{p, X}}{\longrightarrow} \sim \mathrm{O}^{*} \\
& \eta^{*}+0 \stackrel{P_{r}^{p, X}}{\longrightarrow} \sim \mathrm{S}^{*} \\
& \sim \mathrm{O}^{*}+\stackrel{P_{r}^{p, P}}{\longrightarrow} \sim \mathrm{S}^{*} \\
& 2 \xi+3 \stackrel{P_{r}^{p, P}}{\longrightarrow} \sim 2-\delta^{2}
\end{aligned}
$$<smiles>[O][Mg]O[Mg]</smiles>

\begin{tabular}{|l|c|c|c|}
\hline & Unreacted & Reacted & Active \\
\hline Initiator & $\bigcirc$ & $\bigcirc$ & $\bigcirc^{\star}$ \\
\hline Monomer & $\bigcirc$ & $\bigcirc$ & $\bigcirc^{\star}$ \\
\hline Cross-linker & & & $\bigcirc^{\star}$ \\
\hline
\end{tabular}

Figure 1 

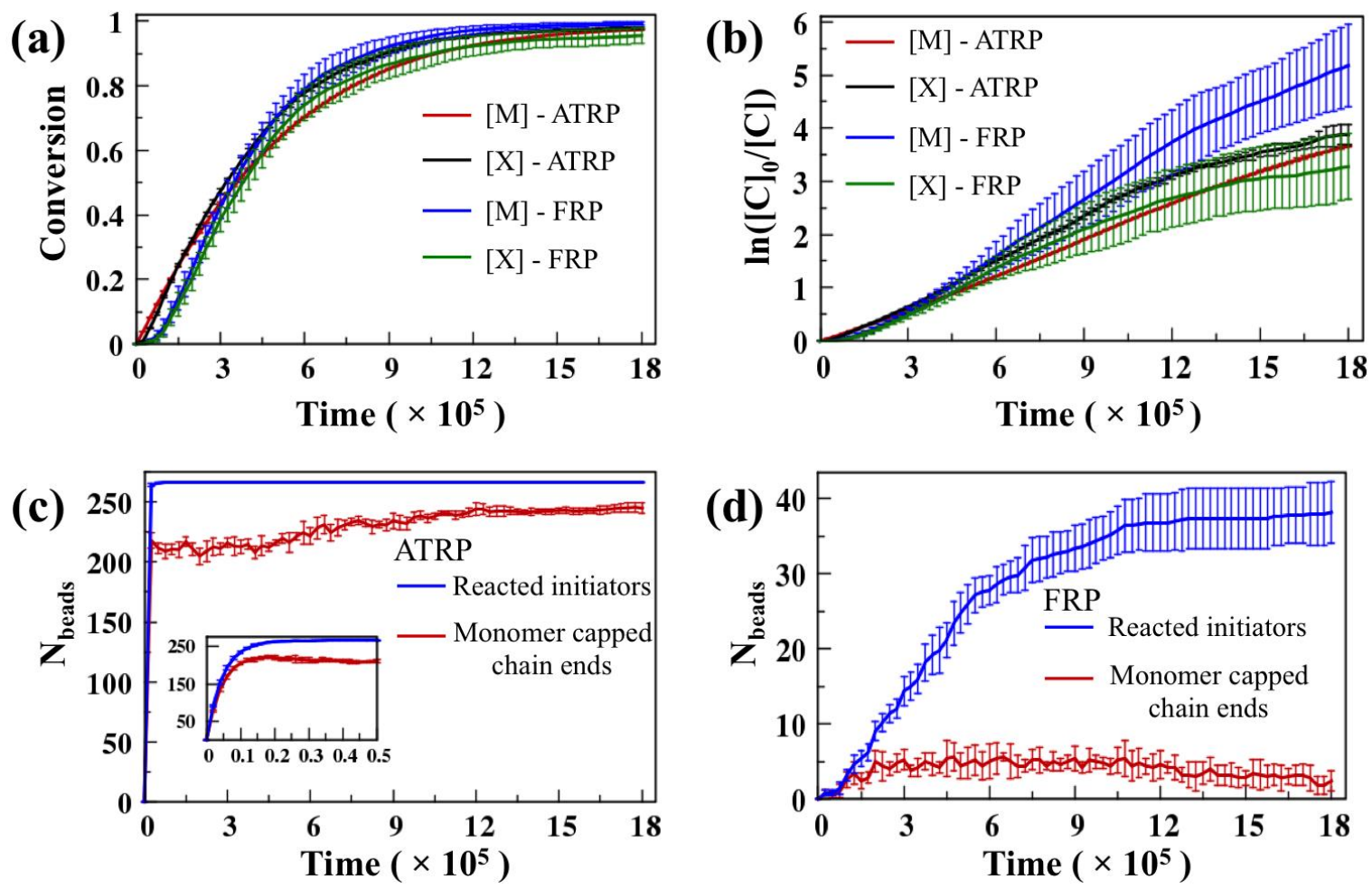

Figure 2 

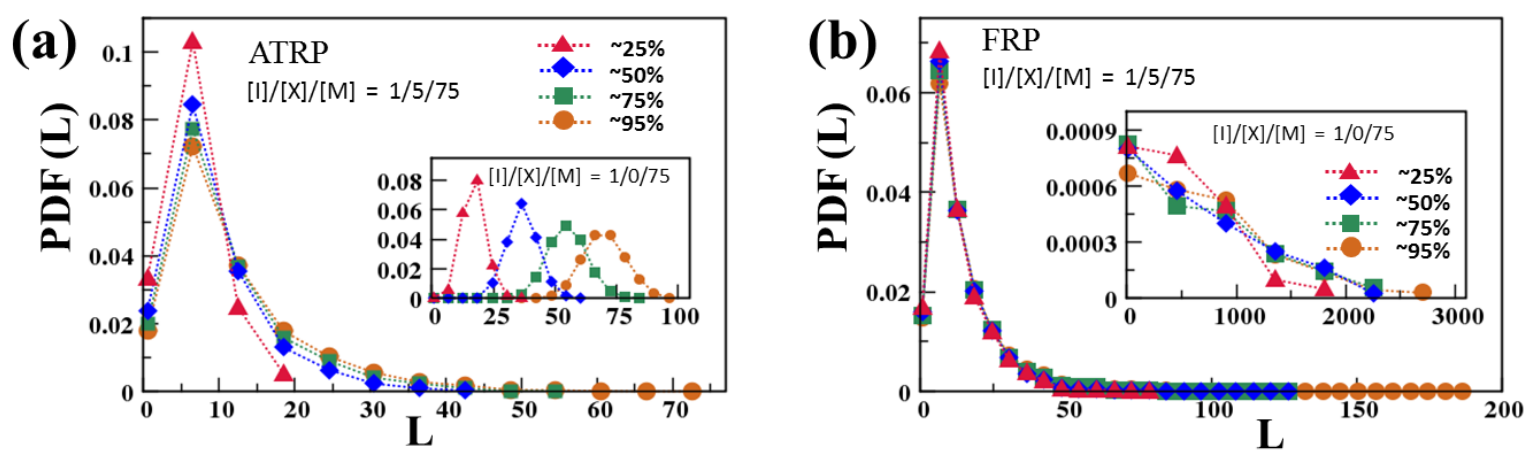

Figure 3 

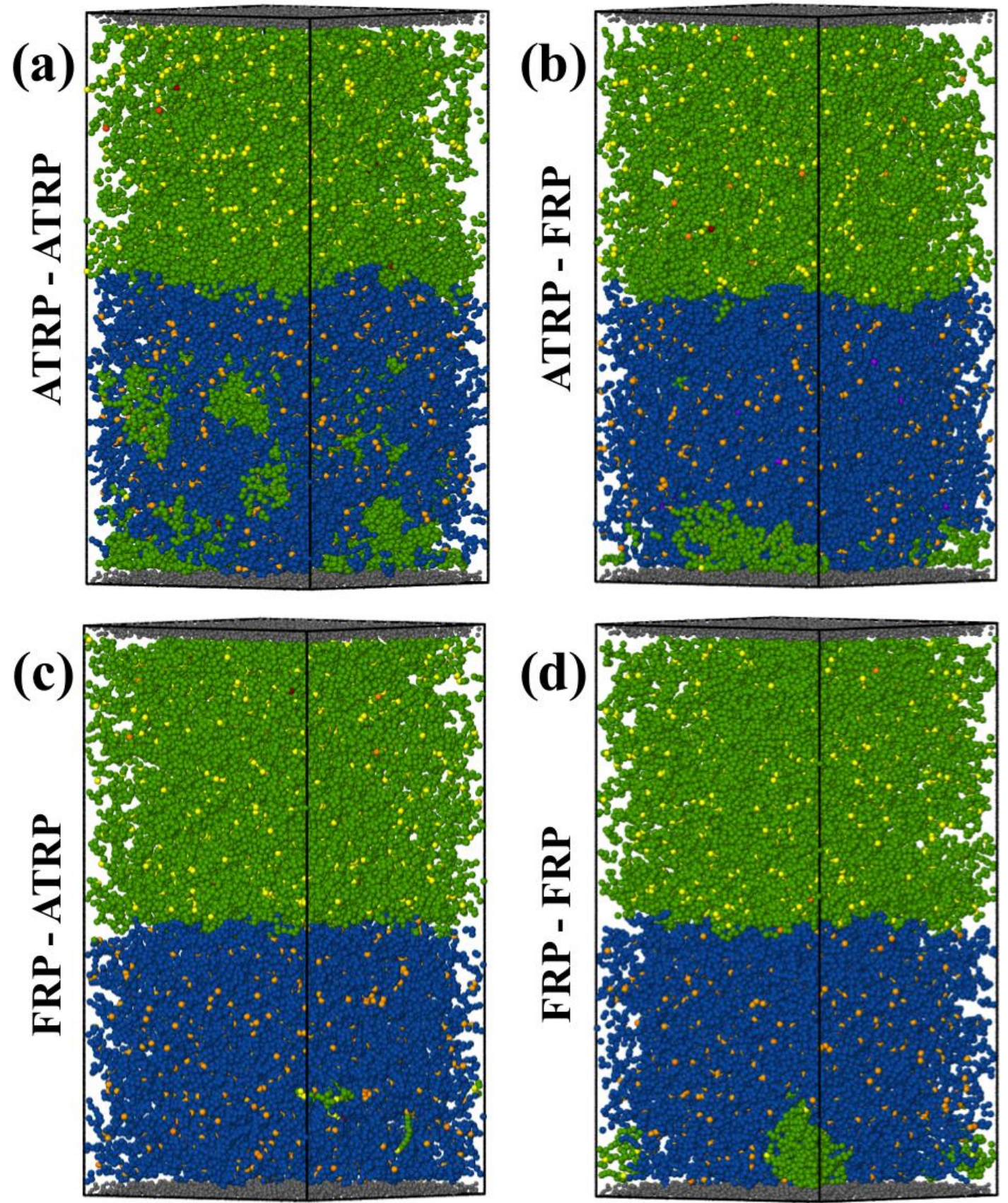

Figure 4 

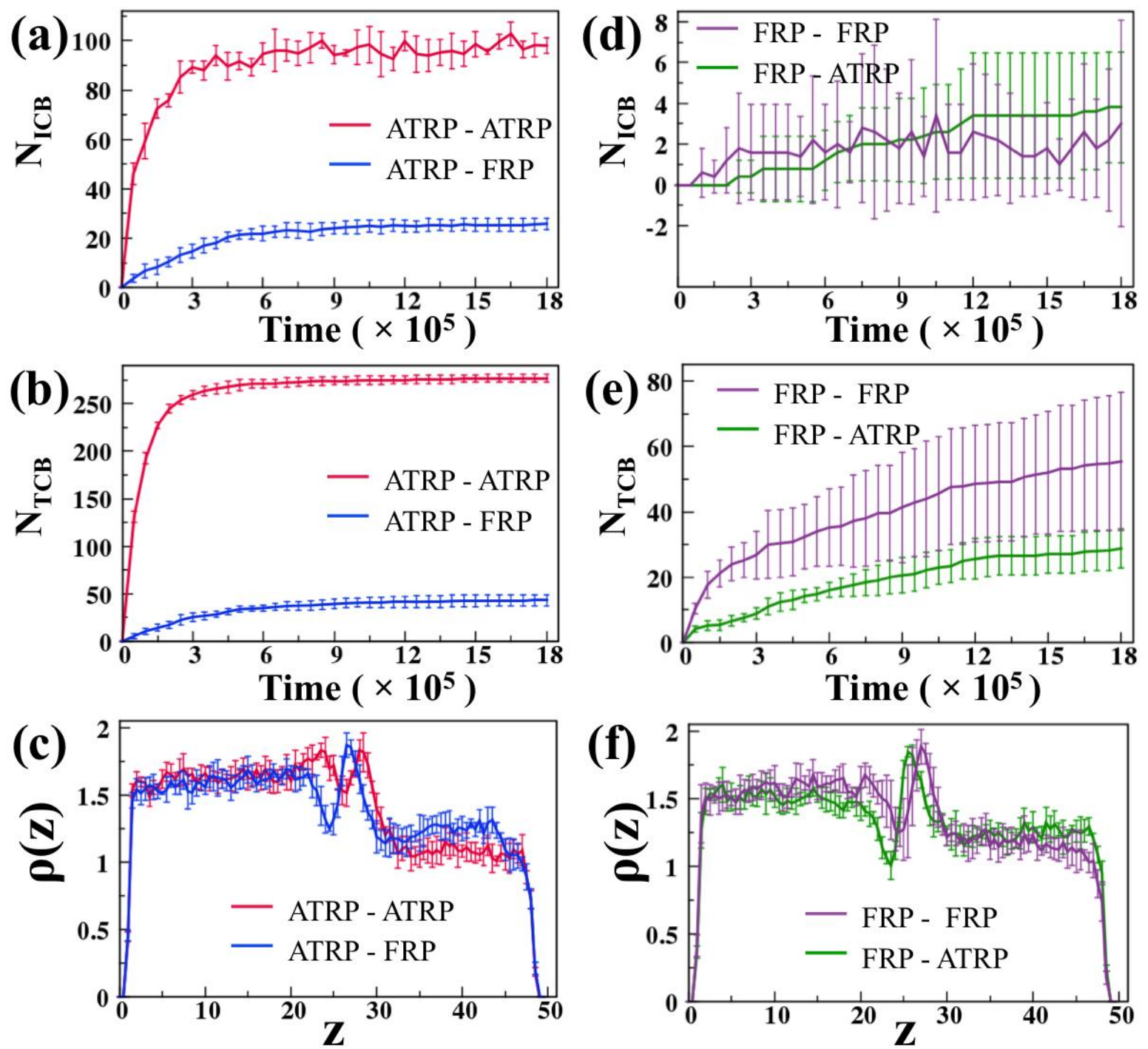

Figure 5 


\begin{tabular}{|c|c|}
\hline Components & Parameter $a_{i j}$ \\
\hline $\mathrm{M} 1-\mathrm{S} 1$ & 25 \\
\hline $\mathrm{M} 2-\mathrm{S} 2$ & 25 \\
\hline $\mathrm{S} 1-\mathrm{S} 2$ & 60 \\
\hline $\mathrm{M} 1-\mathrm{S} 2$ & 60 \\
\hline $\mathrm{M} 2-\mathrm{S} 1$ & 25 \\
\hline $\mathrm{M} 1-\mathrm{M} 2$ & 35 \\
\hline
\end{tabular}

Table 1 


\begin{tabular}{|c|c|c|}
\hline Probability & ATRP in gel1/gel2 & FRP in gel1/gel2 \\
\hline$P_{r}^{i}$ & $2.0 \times 10^{-3}$ & $3.0 \times 10^{-6}$ \\
\hline$P_{r}^{p, M}$ & $2.0 \times 10^{-3}$ & 0.2 \\
\hline$P_{r}^{p, X}$ & $4.0 \times 10^{-3}$ & 0.4 \\
\hline$P_{r}^{p, P}$ & $6.0 \times 10^{-3}$ & 0.6 \\
\hline$P_{r}^{t}$ & - & 1.0 \\
\hline
\end{tabular}

Table 2 
ATRP

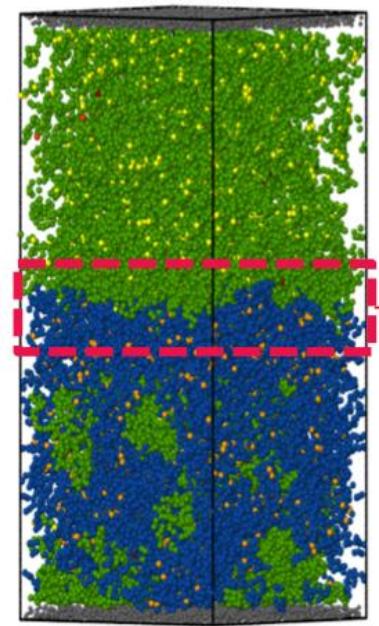

ATRP
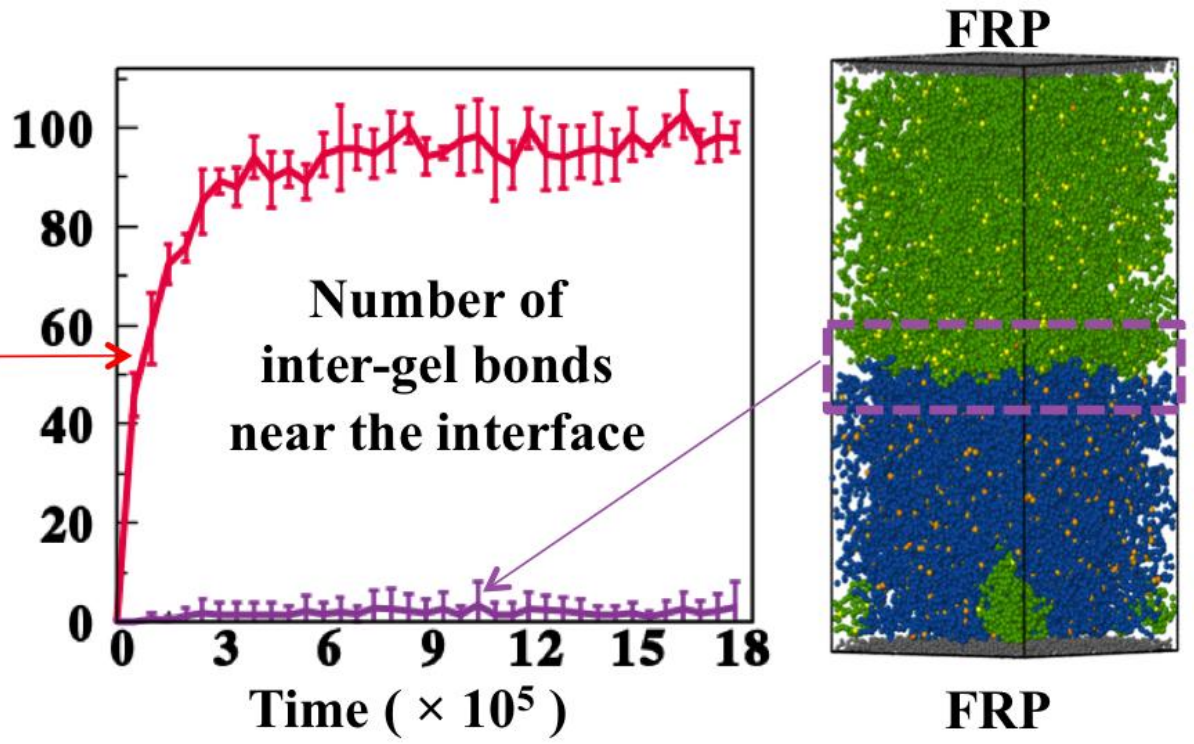